\title{
Can prophylactic cranial irradiation of extensive-stage small cell lung cancer prolong survival?
}

\author{
Hideyuki Harada \\ Division of Radiation Therapy, Radiation and Proton Therapy Center, Shizuoka Cancer Center, Shizuoka, Japan \\ Correspondence to: Hideyuki Harada, MD, PhD. Division of Radiation Therapy, Radiation and Proton Therapy Center, Shizuoka Cancer Center, \\ Nagaizumi, Shizuoka, Japan. Email: h.harada@scchr.jp. \\ Comment on: Bang A, Kendal WS, Laurie SA, et al. Prophylactic Cranial Irradiation in Extensive Stage Small Cell Lung Cancer: Outcomes at a \\ Comprehensive Cancer Centre. Int J Radiat Oncol Biol Phys 2018;101:1133-40.
}

Received: 05 December 2018; Accepted: 25 December 2018; Published: 27 December 2018.

doi: $10.21037 /$ tro.2018.12.07

View this article at: http://dx.doi.org/10.21037/tro.2018.12.07

\section{Introduction}

Approximately $10 \%$ to $15 \%$ of lung cancers are small cell lung cancers (SCLCs). SCLC is divided into two subpopulations-limited-stage (LS) SCLC and extendedstage (ES) SCLC. In LS SCLC, all the lesions are inside the ipsilateral thorax. Lesions beyond this site are categorized as ES SCLC. Prophylactic cranial irradiation (PCI) is considered as the standard of care in patients with LS SCLC who achieve complete response after initial chemotherapy or thoracic radiotherapy. In SCLC, the brain is a common site of distant metastasis. The standard of care for ES SCLC is platinum-based chemotherapy such as cisplatin and etoposide, or cisplatin and irinotecan. Owing to the bloodbrain-barrier, the penetration of cytotoxic chemotherapeutic agents into the brain is prevented. However, radiation can be delivered to the brain, and SCLC is radiosensitive. Thus, several trials have investigated prophylactic cranial irradiation in SCLC. Usually, PCI includes 20 Gy in 5 to 8 fractions, 24 Gy in 12 fractions, 25 Gy in 10 fractions, or $30 \mathrm{~Gy}$ in 10 or 12 fractions. Moreover, an international consortium, including the European Organisation for Research and Treatment of Cancer (EORTC) and Radiation Therapy Oncology Group (RTOG), conducted a phase III trial of dose-fractionation regimens for LS SCLC. This trial compared the standard dose (25 Gy in 10 fractions) with a high PCI dose (36 Gy). No significant difference in the 2-year incidence of brain metastases was found between the two arms, but the 2-year overall survival (OS) rate was $42 \%$ in the standard-dose group and $37 \%$ in the higherdose group ( $\mathrm{P}=0.05)$ (1). On the basis of these data, $25 \mathrm{~Gy}$ in 10 fractions or an equivalent dose-fraction schedule is considered the standard PCI dose for SCLC.

Meta-analyses revealed that PCI decreased the incidence of brain metastases, which translated into an improvement in patient survival after achieving complete response to initial chemotherapy or chemoradiotherapy (2). This analysis included $15 \%$ of ES SCLC cases; however, the benefit of PCI for ES SCLC was borderline from the subset analysis in the previous study. Therefore, the indication of PCI for ES SCLC has been controversial.

On the basis of this background, two large randomized controlled trials for evaluating the significance of prophylactic cranial irradiation of ES SCLC were conducted-one an EORTC study (3) and the other a Japanese study (4). Although the EORTC study reported that PCI improved OS, the Japanese study reported no survival advantage of PCI.

\section{EORTC study}

EORTC conducted a phase III randomized controlled trial that compared PCI with observation in ES SCLC. The major inclusion criteria were age of 18 to 75 years; a performance status of 0 to 2 and ES SCLC before the start of chemotherapy; no progression after four to six cycles of initial chemotherapy; PCI after initial chemotherapy demonstrated improved OS with PCI (median OS, $6.7 v s$. 5.4 months; $\mathrm{P}=0.003$ ); and $\mathrm{PCI}$ judged by investigators as the standard of care for patients with ES SCLC that showed no progression after first-line chemotherapy. 


\section{Japanese study}

Japanese investigators conducted a similar randomized phase III trial in ES SCLC that compared PCI with observation with active magnetic resonance imaging (MRI) surveillance. The major inclusion criteria were ES SCLC before the start of initial chemotherapy; age of $\geq 20$ years; a performance status of 0 to 2 ; no progression after completion of two or more cycles of initial platinumbased doublet chemotherapy; and absence of brain metastases confirmed on gadolinium-enhanced MRI. Surveillance MRIs were also mandatory in the Japanese study. This study demonstrated no significant difference in progression-free survival and a trend toward deteriorated OS with PCI (median OS, 13.7 vs. 11.6 months; $\mathrm{P}=0.09$ ).

\section{Study by Bang et al.}

Bang et al. conducted a retrospective review of patients with ES SCLC who were treated in their institution (5). They reviewed the medical records of 397 consecutive patients with ES SCLC, and 155 patients without baseline brain metastases who had at least a partial response to chemotherapy were selected for the analysis. The patients were stratified according to whether they underwent initial post-chemotherapy brain imaging. However, as the authors described, follow-up or surveillance brain imaging was not obtained unless any symptoms of brain metastasis were suspected. They analyzed OS, time to brain metastasis, and prognostic factors affecting OS. The authors reported that PCI improved OS [hazard ratio (HR), $0.55 ; 95 \%$ confidence interval (CI), 0.39-0.77; $\mathrm{P}=0.0005]$ and time to brain metastasis (HR, 0.40; 95\% CI, 0.23-0.66; $\mathrm{P}=0.0004)$. The median survival in the PCI and non-PCI groups was 13.5 and 8.5 months, respectively. A survival benefit with PCI was observed in patients who underwent post-chemotherapy brain imaging as well as in those who did not. Other statistically significant prognostic factors of OS were the presence of extra-thoracic metastases and performance status.

\section{Discussion}

Both large randomized studies revealed the reduction of brain recurrence in the PCI arm. However, the EORTC study revealed a survival benefit with PCI, but not the Japanese study. Several points are controversial. First, the differences between the two large randomized trials must be identified. The EORTC study did not perform brain imaging at randomization and follow-up unless symptoms were suggestive of brain metastases. In a previous study, $15 \%$ of patients with SCLC had asymptomatic brain metastases on MRI at diagnosis (6). As the authors mentioned, asymptomatic brain metastases might be treated with PCI and may lead to better survival in the PCI arm of the EORTC study.

In the Japanese study, brain imaging before randomization and surveillance imaging were mandatory. Therefore, patients with asymptomatic brain metastasis before study enrollment were excluded from the study. The Japanese study also showed a reduction of brain metastasis by PCI, but this did not translate to progression-free survival and OS. In the Japanese study, $69 \%$ of the patients in the observational arm had newly developed brain metastases, among which $83 \%$ were treated with radiotherapy. This is probably the main reason why survival in the observational arm did not have a negative impact on survival.

The current study by Bang et al. was conducted as a single-institution retrospective study of ES SCLC and demonstrated better survival in patients who underwent PCI after first-line chemotherapy without progression. This study excluded patients who did not receive chemotherapy, showed no response to chemotherapy, and had brain metastases at diagnosis or the development of brain metastases while on chemotherapy, like in the Japanese study. However, surveillance brain imaging was not obtained in the authors' clinical practice, which is different from what was done in the Japanese study. Even when limited to patients who underwent brain imaging at the completion of first-line chemotherapy, the study by Bang et al. showed that PCI had a positive impact on survival, which is different from the finding in the Japanese study.

However, this study has some limitations owing to its retrospective cohort. First, the selection of patients for the two arms was biased. The decision of performing PCI was made according to the discretion of the treating physician. A higher rate of complete response was observed in the PCI group $(19.1 \%$ vs. $1.2 \%, \mathrm{P}<0.01)$. The patients who actually underwent PCI might have maintained good general conditions with good response to first-line chemotherapy. Patients with no visceral or active metastasis after first-line chemotherapy would benefit from PCI.

Second, no description was provided as to the secondline chemotherapy after recurrence. The Japanese study reported higher rates of second-line chemotherapy $(89 \%$ vs. 59\%) than the EORTC study. The study by Bang et al. lacks information on second-line chemotherapy; thus, we 
could not judge the effect of second-line chemotherapy. Moreover, recent clinical trials of second-line chemotherapy reported promising a median survival range of 8 to 18 months $(7,8)$. That is, no second-line chemotherapy after progression means limited patient life expectancy due to visceral dissemination. In this situation, PCI may be the only antitumor therapy, which would translate to prolonged survival. If both the patient and the physician choose an aggressive strategy like in the Japanese study, second-line chemotherapy would suppress visceral dissemination, but brain metastasis could be detected during a prolonged lifetime by surveillance brain imaging, which could be treated with radiotherapy. PCI itself is potentially toxic to cognitive function as the authors mentioned. A cognitive analysis of RTOG trials 0212 and 0214 showed a higher rate of decline of cognitive function in the PCI arm than in the non-PCI arm (9).

PCI may also impact a negative general condition. In the EORTC study, $12.5 \%$ more patients in the PCI group experienced severe worsening of global health status 3 months after PCI (10). A PCI-preserving strategy could avoid these toxicities until new brain metastases are detected on surveillance brain imaging.

\section{Conclusions}

In the study by Bang et al. and the two large randomized controlled trials, the therapeutic value of PCI differed according to the medical circumstance in each community and the strategy for each patient. According to the Japanese clinical guideline, PCI is not recommended for our medical situation such as easy access to MRI and high rate of second- or later-line chemotherapy. The decision of performing PCI should be made after considering these factors, with sufficient discussion between the patient and the physician.

\section{Acknowledgments}

The submitted work was supported by grant from the National Cancer Center Research and Development fund. Funding: None.

\section{Footnote}

Provenance and Peer Review: This article was commissioned and reviewed by the Section Editor Hsin Hua Nien (Department of Radiation Oncology, Cathay General
Hospital, Taipei, Taiwan).

Conflicts of Interest: The author has completed the ICMJE uniform disclosure form (available at http://dx.doi. org/10.21037/tro.2018.12.07). Dr. Harada reports personal fees from Daiichi sankyo pharmaceutical co., personal fees from Astrazeneca pharmaceutical co., personal fees from Brainlab co., personal fees from Chugai parmaceutical co. , grants from Japan Agency for Medical Research and Development, grants from The National Cancer Center Research and Development fund, personal fees from Novaltisoharma co., personal fees from Eli Lilly Japan K.K, outside the submitted work.

Ethical Statement: The author is accountable for all aspects of the work in ensuring that questions related to the accuracy or integrity of any part of the work are appropriately investigated and resolved.

Open Access Statement: This is an Open Access article distributed in accordance with the Creative Commons Attribution-NonCommercial-NoDerivs 4.0 International License (CC BY-NC-ND 4.0), which permits the noncommercial replication and distribution of the article with the strict proviso that no changes or edits are made and the original work is properly cited (including links to both the formal publication through the relevant DOI and the license). See: https://creativecommons.org/licenses/by-nc-nd/4.0/.

\section{References}

1. Le Péchoux C, Dunant A, Senan S, et al. Prophylactic Cranial Irradiation (PCI) Collaborative Group. Standarddose versus higher-dose prophylactic cranial irradiation (PCI) in patients with limited-stage small-cell lung cancer in complete remission after chemotherapy and thoracic radiotherapy (PCI 99-01, EORTC 22003-08004, RTOG 0212, and IFCT 99-01): a randomised clinical trial. Lancet Oncol 2009;10:467-74.

2. Aupérin A, Arriagada R, Pignon JP, et al. Prophylactic cranial irradiation for patients with small-cell lung cancer in complete remission. Prophylactic Cranial Irradiation Overview Collaborative Group. N Engl J Med 1999;341:476-84.

3. Slotman B, Faivre-Finn C, Kramer G, et al. EORTC Radiation Oncology Group and Lung Cancer Group. Prophylactic cranial irradiation in extensive small-cell lung cancer. N Engl J Med 2007;357:664-72. 
4. Takahashi T, Yamanaka T, Seto T, et al. Prophylactic cranial irradiation versus observation in patients with extensive-disease small-cell lung cancer: a multicentre, randomised, open-label, phase 3 trial. Lancet Oncol 2017;18:663-71.

5. Bang A, Kendal WS, Laurie SA, et al. Prophylactic Cranial Irradiation in Extensive Stage Small Cell Lung Cancer: Outcomes at a Comprehensive Cancer Centre. Int J Radiat Oncol Biol Phys 2018;101:1133-40.

6. Hochstenbag MM, Twijnstra A, Wilmink JT, et al. Asymptomatic brain metastases (BM) in small cell lung cancer (SCLC): MR-imaging is useful at initial diagnosis. J Neurooncol 2000;48:243-8.

7. Goto K, Ohe Y, Shibata T, et al. Combined chemotherapy with cisplatin, etoposide, and irinotecan versus topotecan alone as second-line treatment for patients with sensitive relapsed small-cell lung cancer (JCOG0605): a multicentre, open-label, randomised phase 3 trial. Lancet

doi: $10.21037 /$ tro.2018.12.07

Cite this article as: Harada H. Can prophylactic cranial irradiation of extensive-stage small cell lung cancer prolong survival? Ther Radiol Oncol 2018;2:65.
Oncol 2016;17:1147-57.

8. Murakami H, Yamamoto N, Shibata T, et al. A single-arm confirmatory study of amrubicin therapy in patients with refractory small-cell lung cancer: Japan Clinical Oncology Group Study (JCOG0901). Lung Cancer 2014;84:67-72.

9. Gondi V, Paulus R, Bruner DW, et al. Decline in tested and self-reported cognitive functioning after prophylactic cranial irradiation for lung cancer: pooled secondary analysis of Radiation Therapy Oncology Group randomized trials 0212 and 0214. Int J Radiat Oncol Biol Phys 2013;86:656-64.

10. Slotman BJ, Mauer ME, Bottomley A, et al. Prophylactic cranial irradiation in extensive disease small-cell lung cancer: short-term health-related quality of life and patient reported symptoms: results of an international Phase III randomized controlled trial by the EORTC Radiation Oncology and Lung Cancer Groups. J Clin Oncol 2009;27:78-84. 